\title{
Qualities of a good reviewer
}

\section{Huyen Bui ${ }^{1}$, Dallas Dunlap ${ }^{2}$, Thomas Hearon ${ }^{3}$, Donald Herron ${ }^{4}$, Chaoli Lan ${ }^{5}$, Shu Jiang ${ }^{6}$, Kurt Marfurt ${ }^{7}$, Balazs Nemeth ${ }^{8}$, Osareni Ogiesoba ${ }^{2}$, Gerard Schuster ${ }^{9}$, and Hongliu Zeng ${ }^{2}$}

Interpretation shares commonalities with GeOPHYSICS and the $A A P G$ Bulletin in that it is a peer-reviewed journal. Unlike GEOPHYSICS and the AAPG Bulletin, Interpretation is built around special sections headed by a team of special-section editors who are either experts or particularly interested in the focused area. In addition to constructing a Call for Papers announcing their special section, the special-section editors also will solicit papers from colleagues, competitors, technology suppliers, and others that they believe may have contributions of interest to the Interpretation readership community. Submitted papers then are assigned by the special editors to three or more reviewers, many of whom are contributors to (and hence expert in) the same special-section topic. By design, the special section-structure of Interpretation reaches authors, editors, and reviewers who previously may not have been involved in the peer-review process. Recognizing this fact, in this article the standing editorial board attempts to summarize some of the more important qualities of what we find to be a good reviewer.

\section{Expectations of reviewers}

Reviewing a paper takes time. However, the time invested helps not only the authors of the paper but also our profession, our professional societies, and the scientific community at large. The flowchart in Figure 1 summarizes the review process. The expectation is that reviewers will provide a timely, professional review. Reviewers are expected to critique but not correct poor English grammar. Simply circling a sentence or paragraph and writing "rephrase," "unclear," "unsubstantiated," or "garbled" is sufficient. A weak point of many papers, common to new authors but also to those who have not written a peer-reviewed paper in many years, is the linkage to other work. Here, the reviewers should ask the authors to add additional specific references that put their paper in context, or to reference papers that propose an alternative workflow or interpretation. A good reviewer may suggest some related and necessary references beyond that of their work group.

By construction, many, if not most papers submitted to Interpretation are either multidisciplinary or they integrate diverse types of data. As a reviewer, you are not expected to be a content expert for every paper. If a paper has detailed algorithmic derivations in an appendix and you are uncomfortable with such arithmetic, simply tell the Associate Editor that you "leave the review of the equations to those who are more proficient in mathematics" and review the remainder of the paper. Alternatively, if you are comfortable with the mathematics or a detailed seismic processing workflow, but not with the geologic application, tell the editor that you have "reviewed the algorithms and workflows in detail, but leave the interpretation to those more familiar with this type of geologic problem." It is then up to the Associate Editor to choose a cross section of reviewers able to critique the paper in its entirety.

There are multiple formats in constructing and uploading a review. Reviewers who spend much of their lives on the computer or mobile devices may feel it easiest to edit the manuscript in Adobe Writer. Other reviewers like to construct a suite of point-by-point comments using word-processing software and then copy/paste their review in the area provided. Still others will print out a hard copy, cross out paragraphs, draw arrows to move text, add comments in the margin or between lines, and then scan and upload the results. All three of these options are acceptable. Use the review process that is most time efficient for you, the reviewer.

\section{Technical content}

A quality review should assess the correctness of assumptions, conclusions, and theory. Did authors provide details on the limitations of their method and/or results? A good reviewer knows when and how to ask

\footnotetext{
${ }^{1}$ Shell International E\&P, Houston, Texas, USA. E-mail: Huyen.Bui@Shell.com.

${ }^{2}$ Bureau of Economic Geology, Austin, Texas, USA. E-mail: dallas.dunlap@beg.utexas.edu; osareni.ogiesoba@beg.utexas.edu; hongliu.zeng@beg. utexas.edu.

${ }^{3}$ ConocoPhillips, Houston, Texas, USA. E-mail: thomashearon@gmail.com.

${ }^{4}$ Consultant, Sugar Land, Texas, USA. E-mail: dherron7@gmail.com.

${ }^{5}$ China University of Petroleum-Beijing, College of Petroleum Engineering, Beijing, China. E-mail: lanchaoli@163.com.

${ }^{6}$ EGI, Salt Lake City, Utah, USA. E-mail: sjiang@egi.utah.edu.

${ }^{7}$ University of Oklahoma, Norman, Oklahoma, USA. E-mail: kmarfurt@ou.edu.

${ }^{8}$ BHP Billiton Canada Inc., Saskatoon, Saskatchewan, Canada. E-mail: balazs.nemeth@bhpbilliton.com.

${ }^{9}$ KAUST, Jeddah, Saudi Arabia. E-mail: gerard.schuster@kaust.edu.sa.

This piece appears in Interpretation, Vol. 5, No. 3 (August 2017); p. 1A-3A, 1 FIG.

http://dx.doi.org/10.1190/INT-2017-0717-FE.1. @ 2017 Society of Exploration Geophysicists and American Association of Petroleum Geologists. All rights reserved.
} 
questions in an editorial context and asks not only "Why?" but often "Why not?" Be open minded. Having been selected as a reviewer, the Associate Editor has judged that you are familiar with or a practitioner of best practices within the topic of the paper. Do not let that familiarity with the best way of doing things blind you to new innovations. If a proposed workflow is impractical given today's data quality or computational capability, the reviewer should request that the authors state the limitations in their discussion or conclusions. Innovative ideas that may not have commercial use in our profession today, may do so in the future, or may have a major impact in other areas of science and technology.

The journal name Interpretation defines the kind of papers that should be considered for publication. Some papers do describe innovative interpretational workflows or data analysis techniques. Many more describe interpretation and data analysis best practices, while
Figure 1. Flowchart showing the Interpretation review process. (1) The author writes the paper, which in step (2) is submitted to Interpretation using the online submittal website. Once submitted, the paper is (3) evaluated by the Interpretation editorial staff to assure it meets the minimum Interpretation style and format requirements. As part of this process, they submit the paper to iThenticate, which compares the paper to previously published documents. If there is more than $5 \%$ overlap from one paper or more than $20 \%$ overlap from multiple papers, the manuscript is sent to the Editor-in-Chief (EIC). The EIC evaluates the paper for plagiarism and then either passes it on for review or sends it back to the author as not being acceptable for review. $\mathrm{Pa}$ pers that are acceptable for review go to the Assistant Editor (ASE), who examines the expertise and workload and then (5) assigns the paper to one of the Associate Editors (AEs). The associate editor (AE) examines the paper and (6) enlists the aid of three Reviewers. After evaluation, each Reviewer (7) sends their review back to the $\mathrm{AE}$. If the reviews are inconsistent, or if the AE is particularly interested in the paper, the $\mathrm{AE}$ adds an additional review and (8) makes a recommendation to the ASE. The ASE performs the same task as the $\mathrm{AE}$, but evaluates the recommendation and (if added) review of the $\mathrm{AE}$ as well. If the paper needs to go back for minor or moderate revision, the ASE (9a) sends it back to the author. If the paper is accepted or rejected, the ASE (9b) sends the paper to the EIC. In general, the EIC does not add a review but does check for Interpretation style, including citation and formatting of references, formatting of equations, unacceptable use of references in the "Abstract" and "Conclusions" sections, inclusions of color bars, axes labels, and scale bars on figures, and the use of active voice in the "Abstract" section. The EIC (10) then sends the final decision of acceptance or rejection to the author.

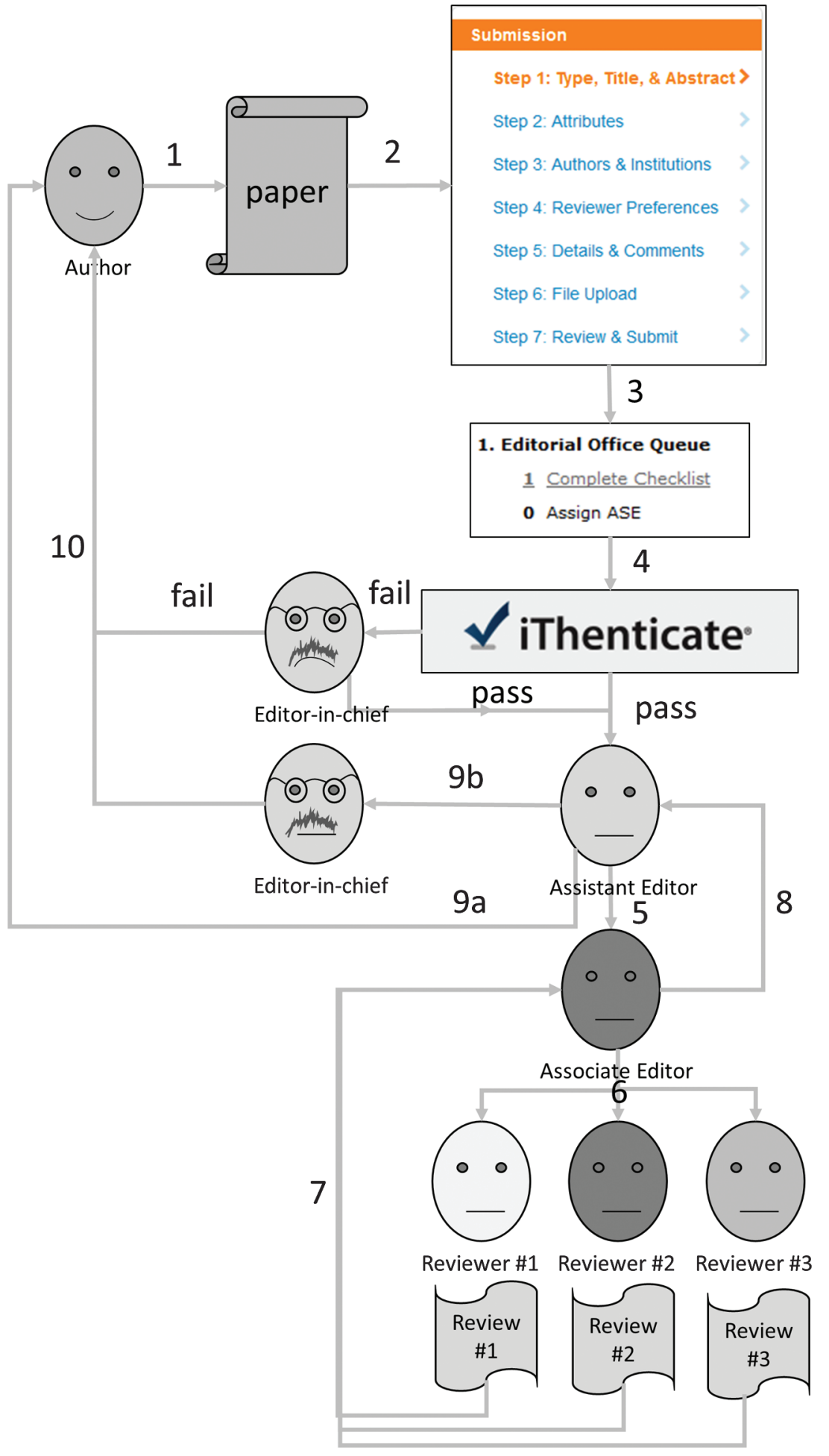


still others describe how such analysis and practices can be applied to a specific geologic or engineering objective. Our readership finds papers on interpretational pitfalls (and how to avoid them) to be of particular interest. The reviewer should ask, "Will this paper add value to the body of scientific knowledge and be of interest to the readership of Interpretation? Does this paper describe geological, geophysical, or engineering phenomena of interest to interpreters?"

\section{Timeliness}

A good review is a timely review. If you do not have the necessary time to review a paper, even if you would like to, decline the invitation and tell the editor whether you would be willing to help out in the future. If you decline, it is good practice to recommend a colleague you think might be interested in performing a review of a paper on this topic. However, after agreeing, sometimes you will receive a paper that has so many grammatical and structural errors that you cannot follow the logic. Do not keep postponing the review. In this case, it is best to inform the Associate Editor that you are unable to review the paper for these reasons. The Associate Editor can then either find someone willing to invest the additional effort to review the paper or send the paper back to the author with the message that the paper needs significant restructuring or grammatical editing.

Own your review. As a reviewer, it is your responsibility to own the review you have posted to a given author, and as such you should be both accountable and responsible for the technical comments you have provided. This means putting your name on the review and not hiding behind the words you have written. Exceptions occur. You may be uncomfortable in rejecting or even critiquing a paper by your boss, key customer, or former professor. Treat all reviews with the same deference. Your review should be polite, courteous, and most important, helpful. Many authors have exerted considerable effort to establish an idea, complete the technical work, draft the figures, and write the manuscript. Carefully worded constructive criticism can help turn a mediocre paper into a great paper. As an author, it is good practice to thank such reviewers in your acknowledgments.

\section{Language issues or writing style?}

Grammatical and spelling errors are sometimes a problem. However, this should not be confused with organizational problems in a paper. Reviewers should provide guidance on to how to better convey thoughts by reorganizing the structure of a paper. Reviewers should discourage writing that is self-promoting as well as self-deprecating in nature. A good reviewer will identify this deficiency and ask the authors to clearly state their contributions in both the "Abstract" and "Conclusions" sections.
In summary, reviewing is similar to coaching. You want the authors (players) to get the most out of their writing effort (athletic performance). A poor coach can cause a player with talent to quit the sport. A good coach can critique a player without discouragement, providing guidance on how to perform in the next round.

\section{Thanks to our reviewers}

The success of a peer-reviewed journal depends on the quality of the volunteer peer reviewers. SEG and AAPG recognize the reviewers who completed at least one review during 2016 and thank them for sharing their time and expertise. A more formal listing of the expectations of reviewers can be found at http://seg.org/ Publications/Policies-and-Permissions/ethical-guidelines under the Reviewers tab.

The current August 2017 issue contains 53 papers. In addition to 14 papers in the general "Technical papers" section, it features the following special sections:

- Computer-assisted seismic interpretation methods; editors: David Johnston, Geoffrey Dorn, Sergey Fomel, Jesse Lomask, Murray Roth, and Tracy Stark; 7 papers published.

- Subsurface expression of igneous systems and their impacts on petroleum systems; editors: Christopher Jackson, Craig Magee, Nick Schofield, Simon Holford, Qiliang Sun, and Stuart Archer; 12 papers published.

- Seismic inversion - Conventional seismic impedance inversion and advanced seismic inversion techniques: Developments, workflow, and case studies; editors: Huyen Bui, Arthur Weglein, Oswaldo Davogustto Cataldo, Sunil Kumar, Scott Singleton, Malleswar Yenugu, Samarjit Chakraborty, and Ramses Meza; 8 papers published.

- Gas hydrates in the South China Sea; editors: Guangfa Zhong, Hongliu Zeng, Shengxiong Yang, Jinqiang Liang, Xuewei Liu, Xin Su, Xiujuan Wang, Changling Liu, and Ming Su; 6 papers published.

- Least-squares migration; editors: Aimee Mao, Gerard Schuster, Kurt Marfurt, Yonghe Sun, Chong Zeng, Bin Wang, Bertrand Duquet, Paul Singer, Wei Dai, Gaurav Dutta, Jerry Young, Yu Zhang, and Michael Kiehn; 3 papers published.

- Skeletonized/sparse/multiscale geophysical inversion for the interpreter; editors: Gaurav Dutta, Amr Ibrahim, Tristan van Leeuwen, Alexander Klokov, and Yunsong Huang; 3 papers published.

Interpretation invites you to contact a member of the Editorial Board or e-mail interpretation@seg.org to submit your ideas for new special sections. 